\title{
High-performance InAs/GaAs quantum-dot laser didoes monolithically grown on silicon for silicon photonics
}

\author{
Mengya Liao, Mingchu Tang, Siming Chen, Jiang Wu, and Huiyun Liu \\ Department of Electronic and Electrical Engineering, University College London, London WC1E 7JE, UK
}

\begin{abstract}
III-V lasers grown on $\mathrm{Si}$ is the most promising solution to light sources on Si platform. The silicon-based telecommunications-wavelength III-V lasers with low threshold current density, high output power, and long lifetime will be presented. As a future driving force technology, Si photonics can improve the lowcost and high speed inter-chip communication by using optical transmission instead of electrical connection. However, Si based light-emitting sources have been considered as "holy grail" for silicon photonics due to Si has in-direct bandgap. By using InAs/GaAs quantum dots (QDs) as active region in laser structure, III-V laser monolithically grown on silicon is a promising solution to be the role of active region on Si platform, which has been proved on GaAs substrate [1,2]. The challenge of monolithic growth is that high density of threading dislocations (TDs) will propagate into laser active region due to the lattice mismatch between GaAs and Si. To stop the TD propagation, defect filter layers (DFLs) formed by InGaAs/GaAs strained-layer superlattices (SLSs) have been applied, which significantly reduce the density of TDs from $\sim 10^{10} / \mathrm{cm}^{2}$ at the interface between III-V and Si to $<10^{6} / \mathrm{cm}^{2}$ in III-V active region $[2,3]$. As a zero-dimensional material, QD has three-dimensional quantum confinements, which create delta function like density of states. QD lasers thus have low threshold currents, temperature insensitive operation, and less sensitivity to threading dislocations. Therefore QD is the ideal candidate to form active region in III-V lasers grown on group IV substrates. High performance QD lasers grown on GaAs substrates have been well-established, which proves its potential of growing on group IV platforms [1].
\end{abstract}

In this presentation, we will review our development of InAs/GaAs QD lasers monolithically grown on $\mathrm{Ge}, \mathrm{Se} / \mathrm{Si}$, and $\mathrm{Si}$ substrates in last few years [2-10]. Low threshold current density of $62.5 \mathrm{~A} / \mathrm{cm}^{2}$, which corresponds to $12.5 \mathrm{~A} / \mathrm{cm}^{2}$ for each of the five QD layers, has been demonstrated under $\mathrm{cw}$ operation at room temperature. The lasing wavelength is at $1315 \mathrm{~nm}$. The output power is as high as $105 \mathrm{~mW}$ at an injection current density of $650 \mathrm{~A} / \mathrm{cm}^{2}$, with no evidence of power saturation up to this current density. The ageing test was performed at a fixed temperature of $26^{\circ} \mathrm{C}$, with the output power monitored for a constant cw drive current of $210 \mathrm{~mA}$. An extrapolated mean time to failure (MTTF) of over 100,158 hours was demonstrated here. These results are a major step towards silicon-based photonics and photonic-electronic integration, and provide a route towards cost-effective monolithic integration of III$\checkmark$ devices on Si platform.

\section{References}

[1] H. Y. Liu, D. T. Childs, T. J. Badcock, K. M. Groom, I. R. Sellers, M. Hopkinson, R. A. Hogg, D. J. Robbins, D. J. Mowbray,

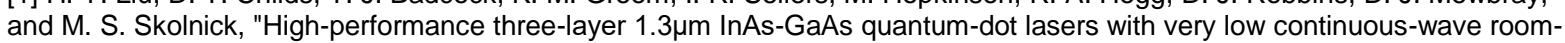
temperature threshold currents," IEEE Photon. Technol. Lett. 17, 1139-1141 (2005).

[2] M. Tang, S. Chen, J. Wu, Q. Jiang, V. G. Dorogan, M. Benamara, Y. I. Mazur, G. J. Salamo, A. Seeds, and H. Liu, "1.3-um InAs/GaAs quantum-dot lasers monolithically grown on Si substrates using InAIAs/GaAs dislocation filter layers," Opt. Express 22, 11528-11535 (2014).

[3] T. Wang, H. Liu, A. Lee, F. Pozzi, and A. Seeds, "1.3um InAs/GaAs quantum-dot lasers monolithically grown on $\mathrm{Si}$ substrates," Opt. Express 19, 11381-11386 (2011).[4] S. Chen, W. Li, J. Wu, Q. Jiang, M. Tang, S. Shutts, S. Elliott , A

Sobiesierski, A. Seeds, I. Ross, P. Smowton, H. Liu, "Electrically pumped continuous-wave III-V quantum dot lasers on silicon, " Nat. Photonics 10, 307-311 (2016)

[4] T. Wang, H. Liu, A. Lee, F. Pozzi, A. Seeds, "1.3- $\mu \mathrm{m}$ InAs/GaAs quantum-dot lasers monolithically grown on Si Substrate", Opt. Express 19, 11381 (2011).

[5] Andrew Lee, Qi Jiang, Mingchu Tang, Alwyn Seeds, and Huiyun Liu, "Continuous-wave InAs/GaAs quantum-dot laser diodes monolithically grown on Si substrate with low threshold current densities", Opt. Express 20, 22181-22187 (2012) [6] A. Lee, Q. Jiang, M. Tang, Y. Zhang, A. J. Seeds, H. Liu, "InAs/GaAs Quantum-Dot Lasers Monolithically Grown on Si, Ge, and Ge-on- Si Substrates", Lee, A.D., Jiang, Q., Tang, M., Zhang, Y., Seeds, A.J. and Liu, H., IEEE J. Sel. Top. Quantum Electron. 19, 1901107 (2013).

[7] S Chen, M Tang, J Wu, Q Jiang, V Dorogan, M Benamara, Y Mazur, G Salamo, AJ Seeds, H Liu, "1.3 um InAs/GaAs quantum-dot laser monolithically grown on Si substrates operating over $100^{\circ} C^{\prime \prime}$, Electron. Lett. 50, 1467-1468 (2014). [8] Tang, M., Wu, J., Chen, S., Jiang Q., Seeds, A., Liu, H., Dorogan, V.G., Benamara, M., Mazur, Y.I., Salamo, G., J. IET Optoelectronics, 61-64 (2015).

[9] Siming Chen, Mingchu Tang, Qi Jiang, Jiang Wu, Vitaliy G Dorogan, Mourad Benamara, Yuriy Mazur, Gregory Salamo, Peter Smowton, Alywn Seeds, Huiyun Liu, "InAs/GaAs Quantum-Dot Superluminescent Light-Emitting Diode Monolithically Grown on a Si Substrate", ACS

Photonics 1, 638-642 (2014)

[10] H. Liu, T. Wang, Q. Jiang, R. Hogg, F. Tutu, F. Pozzi, A. Seeds, "Long-wavelength InAs/GaAs quantum-dot laser diode monolithically grown on Ge substrate", Nat. Photonics 5, 416 (2011) 